\title{
Mixed central and peripheral nervous system disorders in severe SARS-CoV-2 infection
}

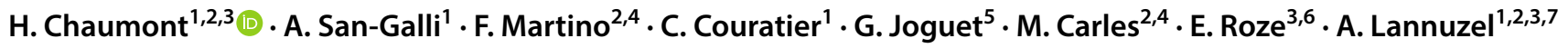

Received: 3 June 2020 / Revised: 5 June 2020 / Accepted: 8 June 2020 / Published online: 12 June 2020

(c) Springer-Verlag GmbH Germany, part of Springer Nature 2020

\section{Dear Sirs,}

We report four cases of severe COVID-19 in male patients aged 50-70 with the combination of central and peripheral nervous system disorders occurring unexpectedly late after the first symptoms. Patients had comorbidities and were admitted for acute respiratory distress syndrome due to a proven SARS-CoV-2 infection. All required mechanical ventilation, among whom one needed an extracorporeal membrane oxygenation support.

Several acute neurological syndromes have been associated with SARS-CoV-2 infection, including anosmia and ageusia [1, 2], meningoencephalitis [3, 4], acute hemorrhagic necrotizing encephalopathy [5], axonal or demyelinating polyradiculoneuropathy [6-8], polyneuritis cranialis

Electronic supplementary material The online version of this article (https://doi.org/10.1007/s00415-020-09986-y) contains supplementary material, which is available to authorized users.

H. Chaumont

hugo.chaumont@chu-guadeloupe.fr

1 Centre Hospitalier Universitaire de la Guadeloupe, Service de Neurologie, 97139 Pointe-à-Pitre/Abymes, France

2 Faculté de Médecine, Université Des Antilles, Pointe-à-Pitre, France

3 Institut National de La Santé Et de La Recherche Médicale, CNRS, Unité Mixte de Recherche (UMR) 7225, Institut du Cerveau Et de La Moelle épinière, ICM, Faculté de Médecine de Sorbonne Université, U 1127, Paris, France

4 Centre Hospitalier Universitaire de La Guadeloupe, Service de Réanimation, Pointe-à-Pitre/Abymes, France

5 Laboratoire de Biologie de La Reproduction, Centre Hospitalier Universitaire de La Guadeloupe, Pointe-à-Pitre/Abymes, France

6 AP-HP, Hôpital de La Pitié-Salpêtrière, Département de Neurologie, Paris, France

7 Centre D'investigation Clinique Antilles Guyane, Inserm CIC 1424, Pointe-à-Pitre, France
[8]. Like in most of the viral infections that involve nervous system, these manifestations occurred within the first ten days after infectious symptoms. Further away from the onset of the disease, when sedation and neuromuscular blocker were withheld, $67 \%$ of the patients with severe COVID-19 develop encephalopathy including prominent agitation, confusion and corticospinal tract signs [9].

In our cases neurological manifestations were detected after mechanical ventilation weaning and extubation (Fig. 1). They consisted of miscellaneous symptoms such as confusion, cognitive dysfunction (memory deficit, frontal syndrome), psychiatric disorders (paranoid delusion, hallucinations), weakness, pyramidal signs, dysautonomia, swallowing dysfunction, vertical supranuclear eye palsy, upper limbs myoclonus, fasciculation and focal muscle atrophy (Table 1). To note, before admission to intensive care unit, patients had no neurological symptom, except for anosmia or ageusia in two of them. One patient had a small acute sub-cortical ischemic stroke on brain MRI. Cerebrospinal fluid (CSF) analysis showed a normal cell count and a moderate increase of protein level in the up to $80 \mathrm{mg} / \mathrm{L}$ in two cases. RT-PCR and IgM for SARS-CoV-2 in the CSF were negative in all patients. On EEG, non-rhythmic frontal slow waves were observed in two patients. Three patients had electrophysiological features of acute motor demyelinating polyradiculoneuropathy with delayed distal latencies and F-waves, slowed conduction velocities and conduction blocks (Supplementary Table). The remaining patient had lower motor neuron features in both the upper and lower limbs. Two patients had an additional decrease of sensorimotor potential amplitude compatible with a critical illness neuropathy. Swallowing and eye movement improved within the first week. Given the persistent muscle weakness and electromyographic features suggesting a post-infectious mechanism, an immunoglobulin therapy was introduced for 5 days. Psychiatric symptoms, cognitive impairment and dysautonomia improved thereafter, but myoclonus and motor weakness of the upper limbs persisted 3 weeks after 


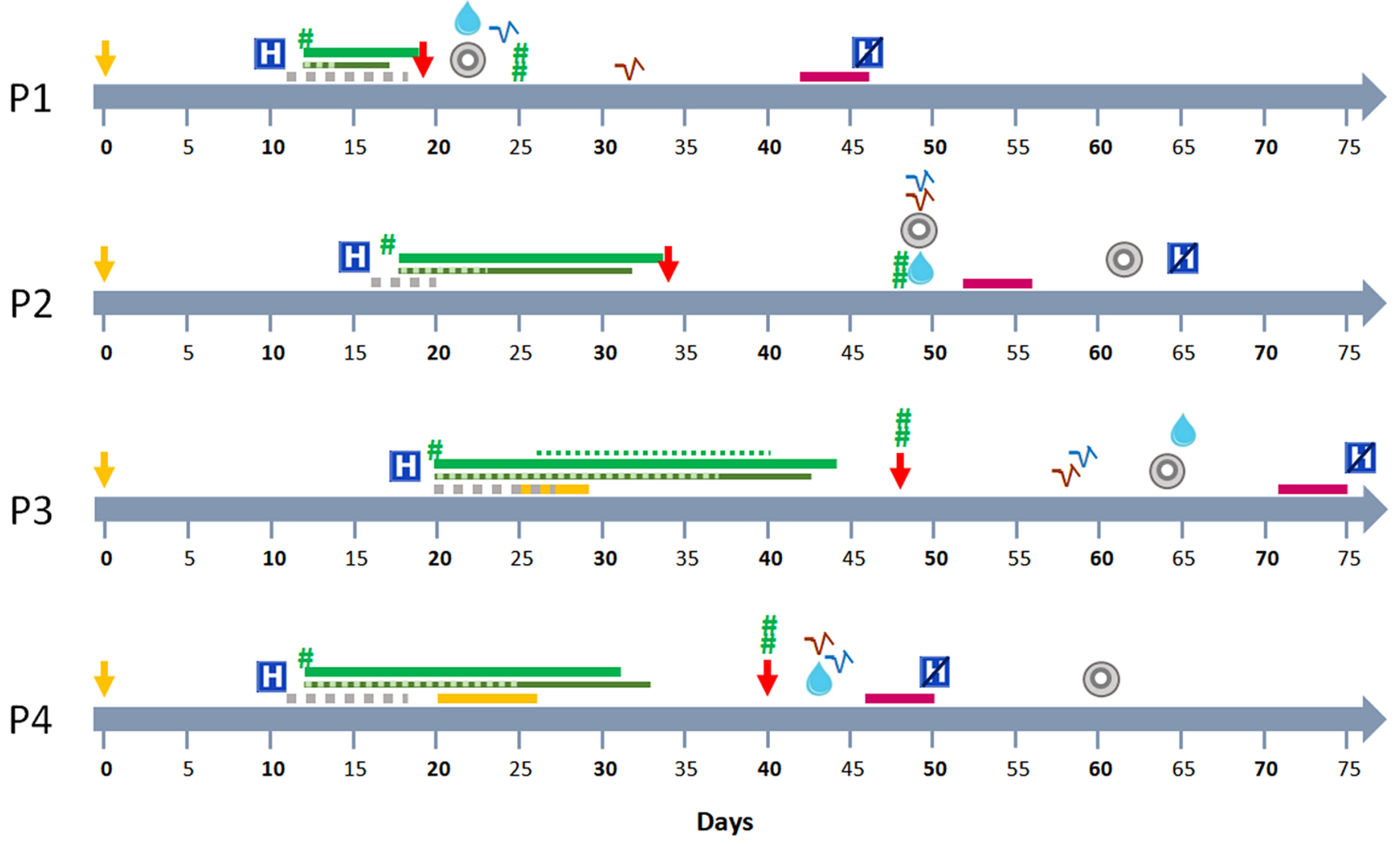

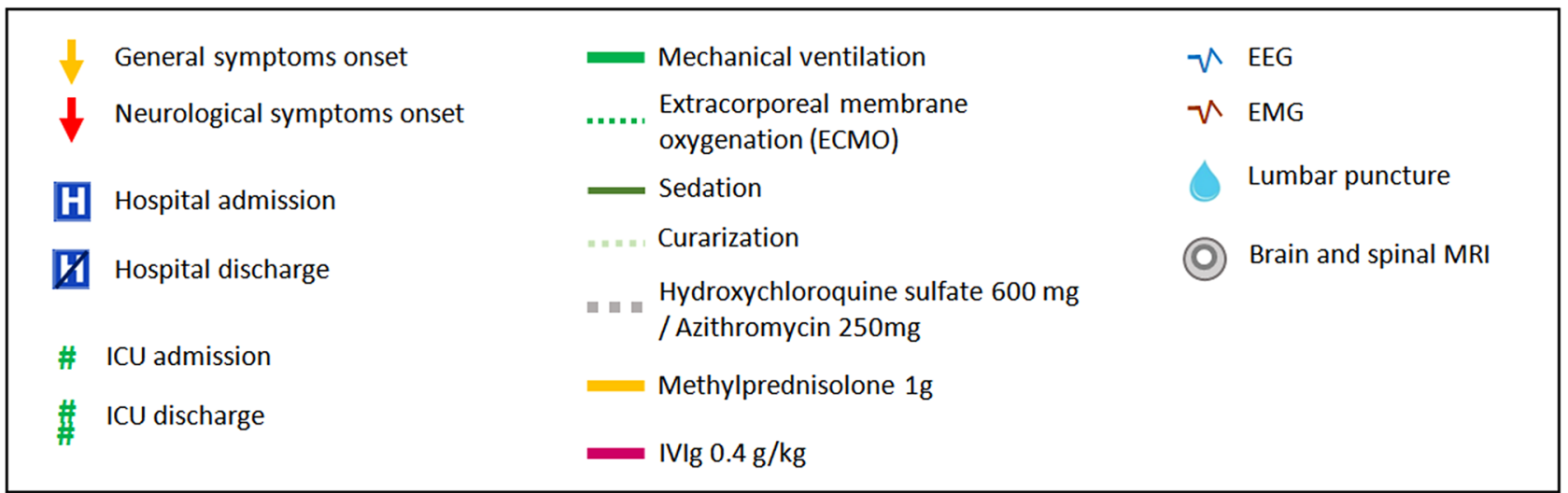

Fig. 1 Timelines showing general and neurological symptoms onset, timing of hospital admission and discharge, timing of ICU admission and discharge, and paraclinical examinations and treatments. $E E G$, electroencephalogram; $E M G$ electromyogram; $I C U$ intensive care unit; IVIg intravenous immunoglobulin; MRI magnetic reso-

discharge. Three patients required prolonged rehabilitation in a specialized center.

We describe here delayed mixed central and peripheral disorders as a complication of severe COVID-19. It combines acute encephalopathy and motor demyelinating polyradiculoneuropathy or diffuse lower motor neuron involvement. Persistent cognitive and motor deficit might result from a critical illness, but neurological features differ from critical illness-related encephalopathy and neuropathy. nance imagery. P1: Patient 1 (M, 62 y.o), P2: Patient 2 (M, 72 y.o), P3: Patient 3 (M, 50 y.o), P4: Patient 4 (M, 66 y.o). For P2, cerebral and spinal MRI were performed at two different dates (days 49 and 62 , respectively)

Critical illness-related neuropathy is characterized by a bilateral, symmetric, axonal sensorimotor polyneuropathy resulting in an areflexic tetraplegia, without dysautonomia or cranial nerves palsy. In our patients, clinical and neurophysiological features of peripheral nervous system involvement could partly reflect critical illness neuropathy but most of them are not expected in this context and are thus more likely linked to COVID-19. Abnormal eye movement, swallowing dysfunction and action myoclonus are unusual in 


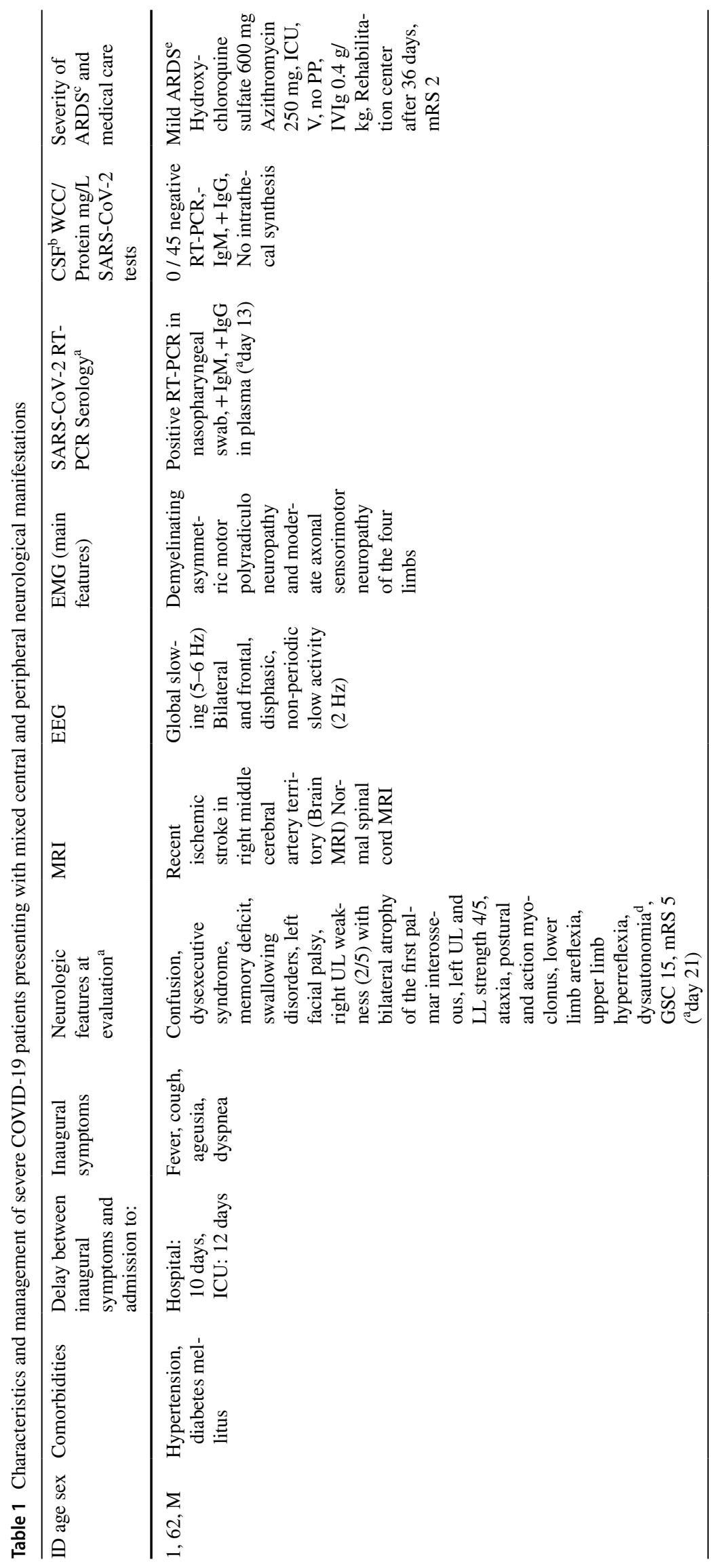




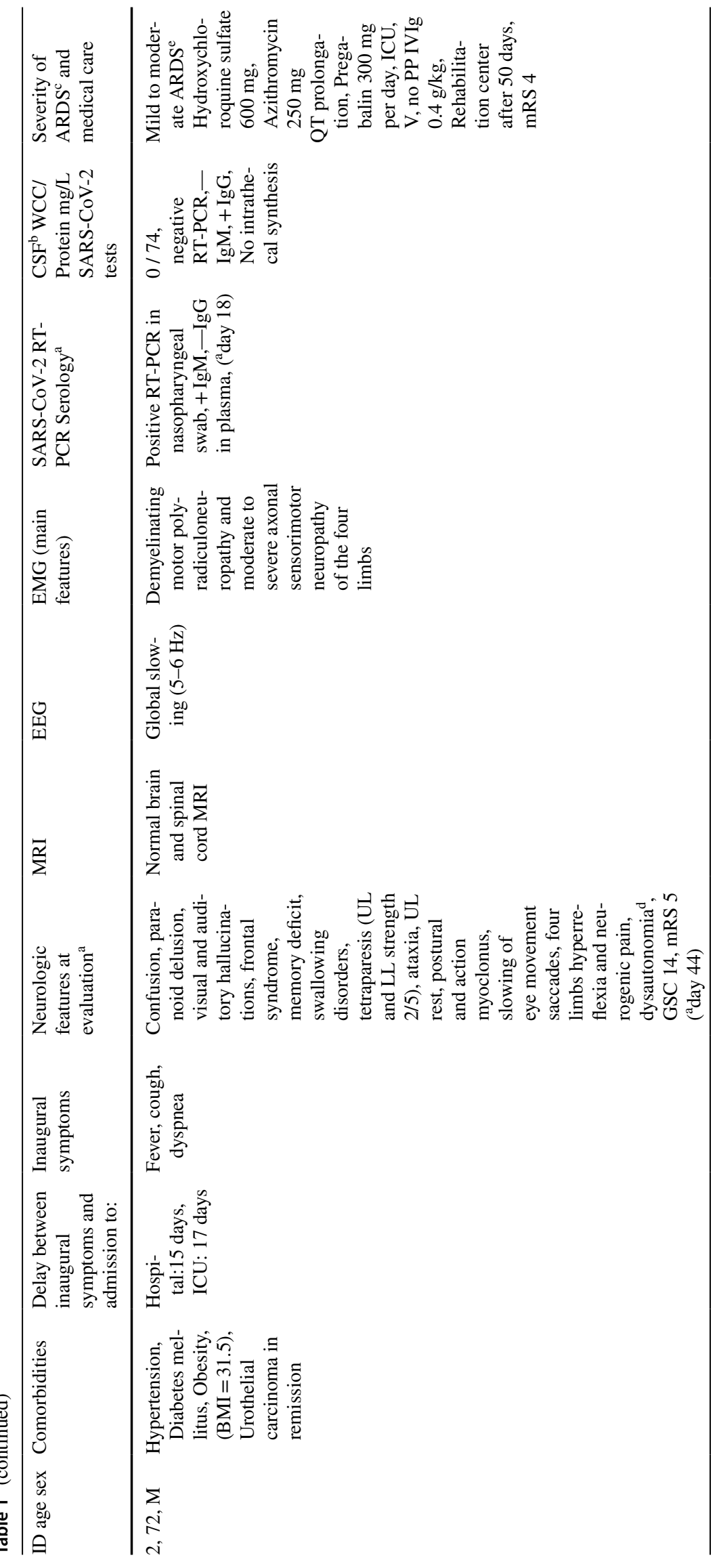




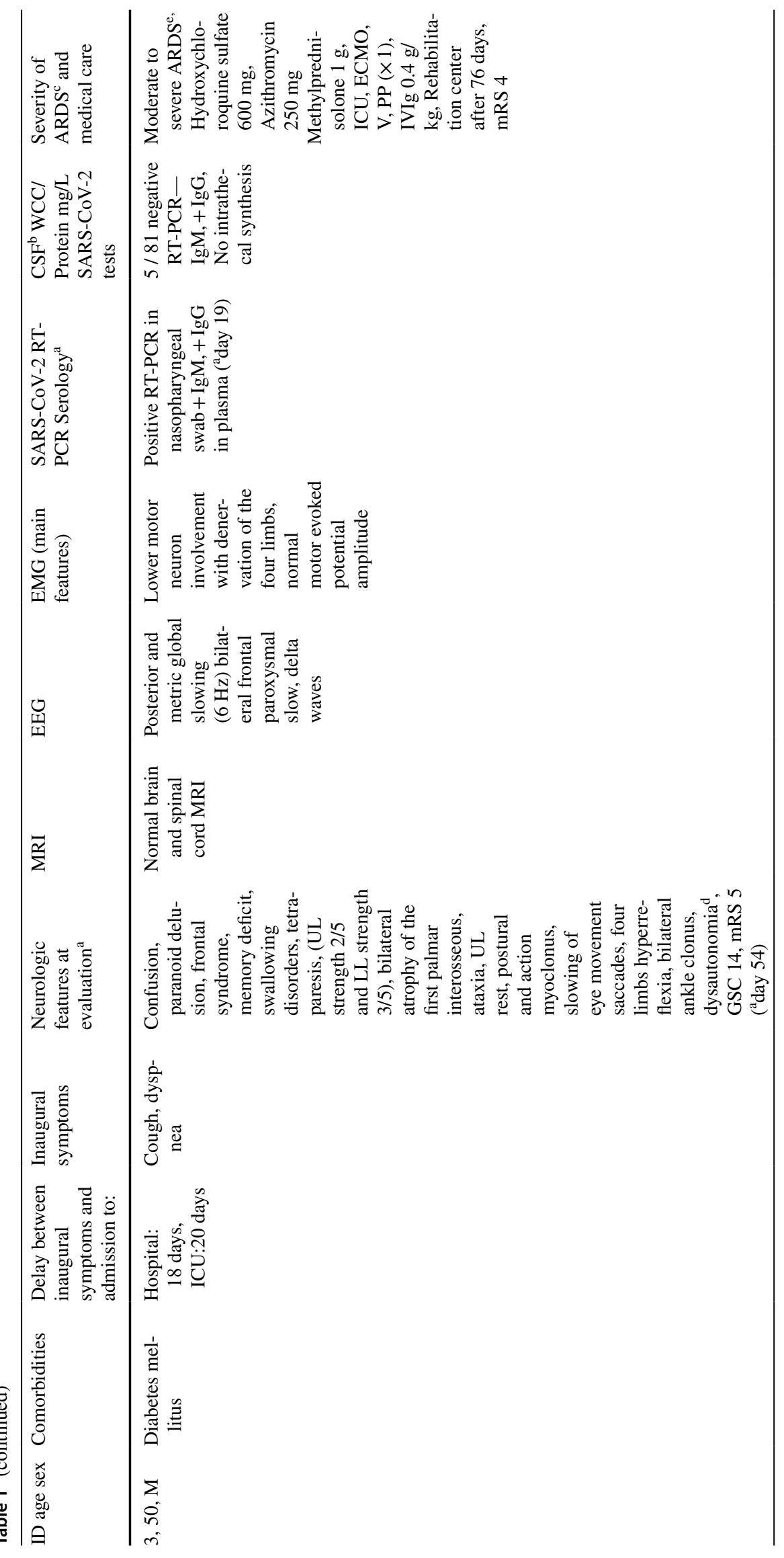




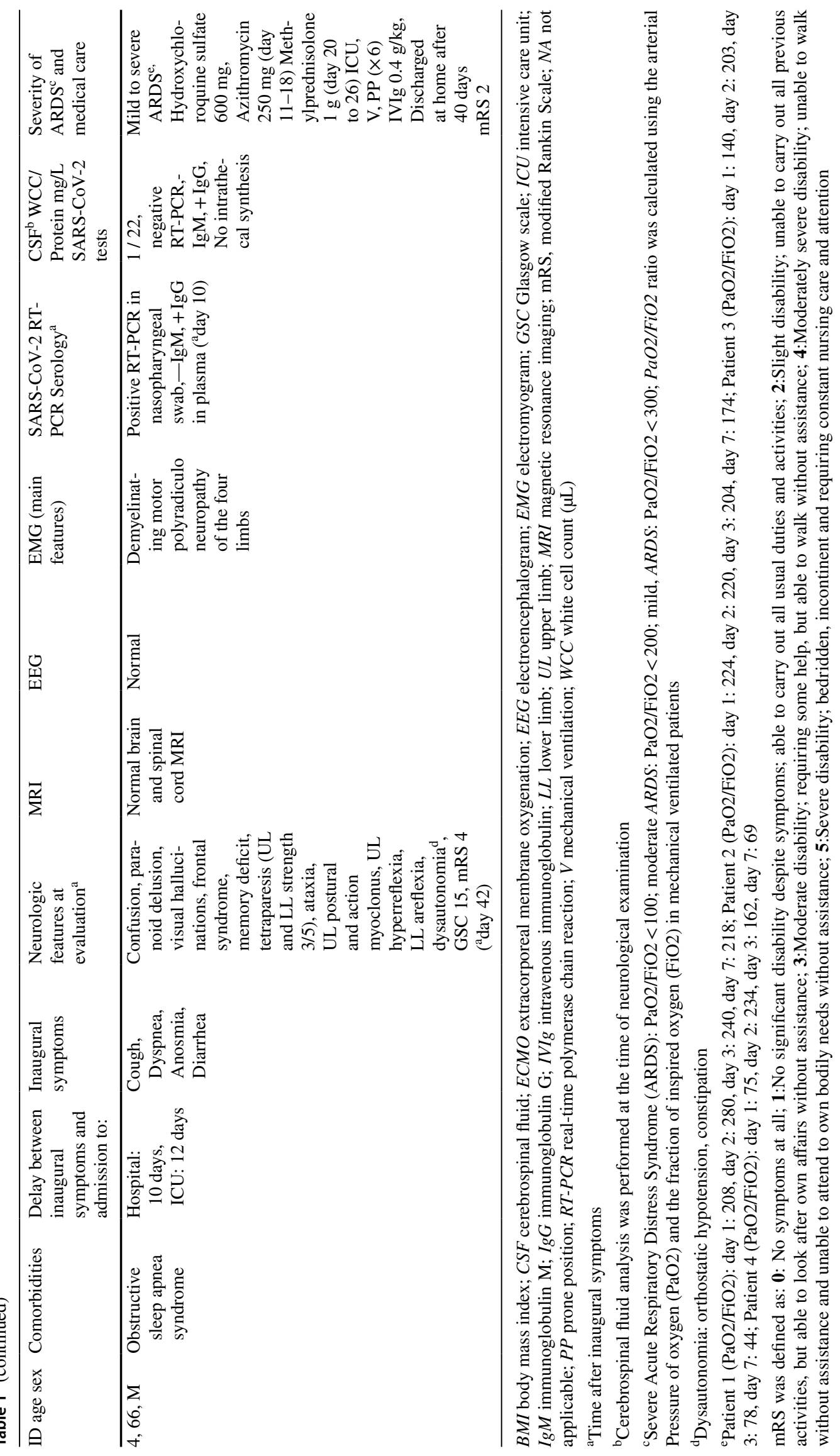


critical illness-related encephalopathy and might rather result from COVID19-related brainstem dysfunction in our patients.

Our study suggests a wider spectrum than previously reported of neurological manifestations associated with COVID-19 and further suggests that patients with severe forms of COVID-19 should be systematically screened for neurological complications.

Acknowledgments The authors thank Yves Chaudière for his language expertise.

Author contributions HC: acquisition, analysis, and interpretation of data and drafting the manuscript; AS-G: acquisition of data and drafting the manuscript; FM: acquisition of data; CC: acquisition of data; GJ: revising the manuscript, analysis, and interpretation of data; MC: acquisition, analysis and interpretation of data and revising the manuscript; ER: drafting and revising the manuscript, analysis, and interpretation of data; AL: acquisition, analysis and interpretation of data, drafting and revising the manuscript.

Funding No funding.

\section{Compliance with ethical standards}

Availability of data and material Not applicable.

Code availability Not applicable.

Consent for publication Patient's consent has been obtained.

Conflicts of interest Dr. Chaumont reports having received travel grant from PEPS development, Roche and Pfizer. Dr. Roze reports served on scientific advisory boards for Orkyn, Aguettant, Merz-Pharma; received honoraria for speeches from Orkyn, Aguettant, Merz-Pharma, Medday-Pharma, Everpharma, International Parkinson and Movement disorders Society; received research support from Merz-Pharma, Orkyn, Aguettant, Elivie, Ipsen, Everpharma, Fondation Desmarest, AMADYS, Fonds de Dotation Brou de Laurière, Agence Nationale de la Recherche; received travel grant from Vitalair, PEPS development, Aguettant, Merz-Pharma, Ipsen, Merck, Orkyn, Elivie, Adelia Medical, Dystonia Medical Research Foundation, International Parkinson and Movement disorders Society, European Academy of Neurology, International Association of Parkinsonism and Related Disorders. Dr. Couratier reports having received travel grant from Allergan, Novartis,
Esai, UCB, Medtronic, Merck Serono, Biogen, LFB, Teva, Icomed, GSK, Genzyme, Aguettant, Cyberonics and Merz-Pharma. Dr. Lannuzel reports having received research support from France Parkinson, PSP France, Agence Nationale de la Recherche, Fonds Européen de DEveloppement Regional, French Ministry of Health, University Hospital of Guadeloupe, received honoraria for a speech from Association des Neurologues du Québec; received travel grant from Vitalair, PEPS development, Merz-Pharma, International Parkinson and Movement disorders Society. Dr. San-Galli, Dr. Martino, Dr. Carles, and Dr. Joguet report no disclosures.

Ethics approval Not applicable.

Informed consent Not applicable.

\section{References}

1. Mao L, Jin H, Wang M et al (2020) Neurologic manifestations of hospitalized patients with coronavirus disease 2019 in Wuhan. JAMA Neurol, China

2. Politi LS, Salsano E, Grimaldi M (2020) Magnetic resonance imaging alteration of the brain in a patient with coronavirus disease 2019 (covid-19) and anosmia. JAMA Neurol. https://doi. org/10.1001/jamaneurol.2020.2125

3. Moriguchi T, Harii N, Goto J et al (2020) A first case of meningitis/encephalitis associated with SARS-Coronavirus-2. Intern $\mathbf{J}$ Diseases 12:19-71. https://doi.org/10.1016/j.ijid.2020.03.062

4. Chaumont H, Etienne P, Roze E et al (2020) Acute meningoencephalitis in a patient with COVID-19. Revue Neurol 35(3):7-87. https://doi.org/10.1016/j.neurol.2020.04.014

5. Poyiadji N, Shahin G, Noujaim D et al (2020) COVID-19-associated acute hemorrhagic necrotizing encephalopathy: CT and MRI features. Radiology 20:11-87. https://doi.org/10.1148/radio 1.2020201187

6. Toscano G, Palmerini F, Ravaglia S et al (2020) Guillain-Barré syndrome associated with SARS-CoV-2. N Engl J Med NEJMc 20:9-91. https://doi.org/10.1056/NEJMc2009191

7. Zhao H, Shen D, Zhou H et al (2020) Guillain-Barré syndrome associated with SARS-CoV-2 infection: causality or coincidence? Lancet Neurol. https://doi.org/10.1016/S1474-4422(20)30109-5

8. Gutiérrez-Ortiz C, Méndez A, Rodrigo-Rey S et al (2020) Miller Fisher Syndrome and polyneuritis cranialis in COVID-19. Neurology 20:20-597. https://doi.org/10.1212/WNL.000000000000961 9

9. Helms J, Kremer S, Merdji H et al (2020) Neurologic features in severe SARS-CoV-2 infection. N Engl J Med NEJMc 20:20-597. https://doi.org/10.1056/NEJMc2008597 